\title{
Cardio-Nephrology: The need for more collaborative efforts between therapeutic clinical trials and social health sciences to preclude cardiovascular disease complications in renal patients.
}

\author{
Muhammad SN \\ The Renal Patient Support Group (RPSG), England UK.
}

\begin{abstract}
Cardiology and Nephrology specialties share several elements in the prevention of Cardiovascular Disease (CVD). CVD prevention in patients with CKD (Chronic Kidney Disease) can only really be understood if there are more robust collaborative clinical trials, and social sciences health research between nephrology and cardiology, not only to inform healthcare and best practices, but also to inform healthcare innovation. More than $80 \%$ of renal patients have one or more cardiovascular risk factors. Randomized Controlled Trials (RCTs) and social science research informs that compromised cardiac health is a key source of demise in patients. The aim here is to highlight the need for more robust collaborations between Nephrology, Cardiology and Therapeutic clinical trials, to preclude CVD complications in renal patients. Chemokine receptors are expressed on cell populations, permitting different chemokine to have selective activity. Chemokine are primarily involved in attraction of leukocytes. CVD intervention takes many forms but begins at acknowledging a need for more targeted therapeutics. Haemodialysis aggravates patient prognosis and myocardial function. Health awareness and education is still a much-needed resource for renal patients; more awareness and comprehension of CVD implemented as part of a patient's health plan. Now more than ever there needs to be a closer link between clinical trials between the two medical specialties, and social sciences to inform how best to prevent patients with CKD developing CVD events.
\end{abstract}

Keywords: Chronic kidney disease, Nephrology, Cardiology, Therapeutics, Biomarkers, Chemokine, Cardiovascular disease.

Accepted on November 05, 2018

\section{Introduction}

Cardiology and Nephrology specialties share several entities in the care of patients, including transplantation, obesity (and diabetic complications), hypertension, medication, Cardiovascular Disease (CVD) ischaemia and aspects of clinical pharmacology, including chemokine/cytokine receptors and therapeutic ligands [1]. Chemokines/cytokines and several receptors in cardiac and renal allograft rejection (particularly chemokine receptor $\mathrm{CXCR} 3$ ) also have a roll in directing posttransplant therapeutics [2].

Cardiovascular Disease (CVD) in the form of Coronary Heart Disease (CHD) is the leading cause of death worldwide; 3.8 million men and 3.4 million women die of the disease each year. CHD was responsible for $16.8 \%$ of total deaths in the UK in (2006) which translates to $\sim 94,000$ deaths. Although CHD mortality rates have been decreasing since the late 1970s, due to better management of risk factors, CHD still poses a huge burden on national resources costing the UK £9-billion in (2006) [3-7].

CVD prevention in patients with Chronic Kidney Disease (CKD) can only really be understood if there are more robust collaborative clinical trials, and social sciences health research between nephrology and cardiology not only to inform healthcare and best practices, but also to inform healthcare innovation [3-7]. Some general concerns surrounding therapeutics for patients with CKD in the post transplanted population surround vascular disease, clotting and thrombosis, infection, malignancy and bone disease - there is still a need to identify suitable targeted treatment to preclude post-transplant health complications.

Mortality can be reduced by pharmacological and/or surgical interventions that allow the rapid return of blood flow to the ischaemic zone of the myocardium, (i.e. by reperfusion therapy). Early and successful myocardial reperfusion with the use of thrombolytic therapy or primary Percutaneous Coronary Intervention (PCI) is one of the most effective strategies for reducing size of a myocardial infarct and improving clinical outcome [3,5-6]. Developing novel therapies that further improve the clinical effectiveness of existing strategies for renal-CVD at risk patients presents an attractive therapeutic opportunity [3,5-6].

\section{Aims}

The aim here is to highlight the need for more robust collaborations between nephrology, cardiology and therapeutic clinical trials, to preclude cardiovascular disease complications in renal patients. 
Citation: Muhammad SN. Cardio-Nephrology: The need for more collaborative efforts between therapeutic clinical trials and social health sciences to preclude cardiovascular disease complications in renal patients. J Clin Nephrol Ther 2019;3(1):3-9.

\section{Chronic Kidney Disease (CKD)}

CKD is a major public health concern because there is considerable overlap between CKD, diabetes and Cardiovascular Disease (CVD); the risk of developing CKD increases with age and it costs the UK $£ 1.02$ billion to prevent Acute Kidney Injury (AKI) [8]. There is a higher risk of dying from AKI than Myocardial Infarction (MI), Breast Cancer, Heart Failure and Diabetes. The kidneys are sensitive and intricate; CKD is graded according to severity-once diagnosed, renal disease is irreversible [8]. Clinical interventions and pharmacological regimes can support patient care, but several risk factors interact with the disease.

CKD tends to establish as a secondary complication to obesity, diabetes, CVD, hypertension or smoking. However, CKD prevention has now become a health priority, especially regarding inhibiting early onset Acute Kidney Injury (AKI). CKD is a health complication to obesity, diabetes. CKD has become more prevalent in primary care [9-13].

Renal disease tends to join CVD complications and as a result, the kidneys reduce in functional capacity. Renal function is determined by creatinine; which is a break-down product of creatine phosphate in muscle [8-10]. Individuals with kidney disease tend to have a range of biochemical imbalances, including sodium, potassium, creatinine and an imbalance of filtering component (nephrons) to rid of toxins in the blood. Renal function is determined by how swift creatinine is removed from blood and this is calculated (determined by Glomerular Filtration Rate) which is used to determine abnormalities waste filtration via nephrons of the kidneys. GFR is the flow rate of filtered fluid through the kidneys [8-10].

There are several risk factors that interact with the disease, and patients can also be affected by the socioeconomic status in addition to age, gender, ethnicity, proteinuria, hypertension, serum phosphate, parathyroid hormone levels, and bone metabolism [11]. CKD/End-Stage Renal Disease (ESRD) is continuation of receiving Haemodialysis (HD) (or alternative renal replacement treatments) until receiving a kidney transplant [11-18].

Diabetic kidney disease remains the single most common cause of kidney failure (26.9\%). By 90 days, $66.3 \%$ of patients were on HD, $19.1 \%$ on Peritoneal Dialysis (PD), 9.7\% had a functioning kidney transplant $4.8 \%$ had died or stopped treatment [11-19].

\section{CKD and Cardiovascular Health}

Cardiology practice is an intricate division of medicine owing to one of the most important organs in human physiology-the heart. In turn, CVD is now the most common cause of death in renal patients. It is not news that smoking raises the risk of an acute coronary syndrome [14] research informs the same epidemiological risk factors for coronary artery disease in the general population are prevalent in renal recipients [15]. There is extensive literature on cardiovascular complications postrenal transplantation and prevention $[1,8,11,12,14]$. More than
$75-80 \%$ of renal recipients have one or more cardiovascular risk factors [14].

Conventionally and fundamentally, practices in both nephrology and cardiology have been clinical and there has been an increase in collaborative efforts between specialties [, $8,11,12,14]$. One team conducts an observational study on the impact of obesity on renal allograft [13]. The team's observation informs obesity raises the risk of pre-operative complications such as wound infections, delayed wound healing, hernias and deep vein thrombosis [13]. Transplantation has a variety of obvious advantages, but specific complications are inevitable. In fact, many pretransplant complications relate CVD relevance, and more are becoming endemic in allografted recipients; hypertension and CHD ensure a high perioperative risk in asymptomatic patients [16].

There is some evidence that Coenzyme Q10 (or CoQ10) supplementation may improve renal function and reduce the need for haemodialysis in patients with CKD and subverting CVD events [17-19]. A randomised controlled clinical trial of patients with chronic heart failure (wherein CoQ10 titres were depleted), CoQ10 supplementation reduced the risk of cardiovascular related mortality by $43 \%$ [19].

Research informs CoQ10 decreased after a regeneration diet (RD). HDL-cholesterol decreased in Water Fasting (WF). Total- and LDL-cholesterol decreased after RD. Other biochemical parameters were within reference values [17]. Research also informs that although the importance of CoQ10 has been mainly attributed to its role as an agent for energy transduction in mitochondria, new functions for CoQ10 including anti-inflammatory effects, gene expression regulation and lipid bilayer membranes stabilization have also been highlighted. This explains its involvement in aging and agerelated diseases such as CVD, CKD and neurodegenerative diseases [18].

Clinical trials and epidemiological studies inform that compromised cardiac health is a frequent complication of CKD and the major cause of death in patients [10]. Though normalising renal function through transplantation and releasing patients from Renal Replacement Therapy (RRT) is crucial, this has not dented the impact on the progression of CVD [16].

\section{Targeting Pharmacology and Therapeutics}

Early in inflammation, as the microvascular endothelium becomes activated, chemokines generated by endothelial cells, sub-endothelial tissue, or released after platelet activation bind to the "activated" endothelial surface $[1,2,8]$. Thus, situated chemokines act as directional signals for leukocytes as they roll across the endothelium.

Important adhesion molecule pairs in this process include the selectins (E, L, and $\mathrm{P}$ ), the Ig-like molecules intercellular adhesion molecule-1, vascular cell adhesion molecule-1, and $\beta 2$ and $\beta 1$ integrins (e.g., leukocyte function-associated antigen-1 and very late antigen-4). After firm adhesion, leukocytes undergo spreading, diapedesis, extravasation, and 
migration into interstitial spaces [16]. CVD intervention takes many forms but begins at acknowledging a need for more targeted therapeutics [20,21].

The impact of polypharmacy in patients with CKD has an impact on renal function over time [14-16]. Evidence informs that modelling based on figures in work by [13] suggests that Angiotensin Converting Enzyme Inhibitors (ACEI)/ Angiotensin Receptor Blockers (ARBs) prescribing for patients with hypertension and proteinuria produces a mean annual net saving to the NHS of $£ 470$ per treated patient over a 5 year perspective [14]. This saving arises through reduced CKD, CVD and MI risk [14-22].

Pharmacological doses of glucocorticoids are suppressive at virtually every level of immune and inflammatory responses, including during activation of the innate immune response and in cellular and humoral acquired responses [20,21]. Thus, glucocorticoids suppress cell adhesion migration, macrophage activation antigen presentation, T-cell receptor expression, Tlymphocyte activation, proliferation, differentiation, and cell function, including cytotoxicity, and B-cell function including antibody production [20-22].

\section{Renal Therapeutics}

A TH-1 pattern of cytokines is characterized by production of largely pro-inflammatory IL-2 and Interferon gamma (IFN- $\gamma$ ), with a primarily cellular immune response. A TH-2 pattern of immunity is characterized by production of IL-4 and IL-10 and is associated with a primarily humoral or antibody response [20]. At physiological concentrations, glucocorticoids inhibit TH-1 and enhance TH-2 cytokine production, thus shifting the immune responses from a TH-1 to a TH-2 pattern [20-22]. Catecholamines, adrenaline particularly, have shown to affect TH-1 and TH-2 patterns of cytokine production. Understanding cytokines to direct treatment to prevent CVD risks in renal patients is important because symptoms in renal patients are already pre-exposed.

The haemodialysis regime is intense; causing vascular complications. Haemodialysis aggravates patient prognosis and myocardial function [21]. The dialysis patient is predisposed to stroke and atherosclerosis. Hypertension occurs early during renal disease and by the time of dialysis, $80 \%$ of patients are chronically hypertensive; $50 \%$ of them with Left Ventricular Hypertrophy (LVH). Ischaemic acute MI is probably now the most common cause of non-infective post-transplant death $[16,22,23]$. There is abundant literature associating posttransplant coronary thrombosis in diabetics and it is noteworthy that such recipients are predisposed to have myocardial risk patterns [24-27].

Immunosuppressive agents that have revolutionized transplantation have direct effects on CVD risk factors and contribute, not only to premature CVD, but also to the increased risk of malignant disease and death due to infection [28,29]. CVD and post-transplant diabetes are compounded by the development of obesity and continue to represent a major challenge to healthcare professionals supporting patients with $\mathrm{CKD}$ and the post-transplant population $[13,30]$. Age-and sex- adjusted cardiovascular risk is increased approximately fivefold higher in renal transplant recipients" [28,29].

Long-term immunosuppression therapy (e.g. Cyclosporine or Tacrolimus) and corticosteroid treatment (Prednisolone) almost certainly raise cholesterol and lipid profile, increasing the risk of obesity, diabetes and potentially aggravating the status of the myocardium [31]. Statin-based pharmacotherapy may become commonly prescribed in renal patients [32-35].

\section{Future Interventions}

Evidence informs that tighter control of adverse immunological events during early post-transplant, has reduced graft loss [36-39]. However, there is an increased risk of CVD in patients receiving immunosuppression. Whilst there have been several clinical biomarkers that can be used to detect CVD [40] in high risk patients in-vivo and in-situ, there still needs to be tighter collaborative efforts between specialties, not only to detect disease but also to inform the development of novel therapeutics.

Since the late noughties, evolving role of chemokines and chemokine receptors in renal transplant allograft rejection have been extensively explored by several teams including $[1,2$, 41-43]. Research has informed the importance of tighter cardiology and nephrology collaborative research in posttransplant animal models [10,14,15,28,29,44].

The currently applied strategies largely focus on general modulation of lymphocyte activation and proliferation. Another interesting pathway which has received far less attention is the attraction of Peripheral Blood Mononuclear Cells (PBMC) [45]. This process of PBMC rolling, adhesion, spreading, extravasation and migration along a gradient involves specific chemotactic cytokines, (i.e. chemokines, and corresponding receptors expressed on PBMC). A variety of chemokines and chemokine receptors have been identified to participate in the process of allograft rejection [45].

Physical activity research has also explored exercise and training influences on cytotoxicity, interleukin-1, interleukin-2 and receptor structures [46]. Considering important relevance of chemokine networks on the immune system, therapeutic strategies could either block pro-inflammatory chemokine signalling or stimulate distribution of PBMC into lymph nodes, termed lymphocyte "homing". More research relating therapeutic and clinical trial intervention studies integrating social sciences research to better target treatment in renal patients at risk of CVD are needed.

\section{The Role of Technology}

The use of Information Communication Technology (ICT) and shared decision making is important for most patients [46]. The use of user-friendly ICT services is also important for knowledge and resource acquisition and for integrated care. User friendly ICT services allow the delivery of better understanding, enhancing care quality efficiencies across care providers, to enable better patient outcomes [46]. The Renal Patient Support Group [RPSG] has also been a great asset to 
Citation: Muhammad SN. Cardio-Nephrology: The need for more collaborative efforts between therapeutic clinical trials and social health sciences to preclude cardiovascular disease complications in renal patients. J Clin Nephrol Ther 2019;3(1):3-9.

patients and carers, providing all to have more healthcare involvement widening shared-decision-making process [47].

Technology can be used to optimise the care for suspect/high risk CVD patients. With the rise in web portals, apps and social media technology, the public are engaging with these to assess and manage health risks. The public's use of technology for all aspects of everyday living has become all-encompassing and has led to changes in behaviour and new models to explore CVD risks $[47,48]$

Technology can thus be used to encourage patients to be more actively involved in their care [47]. This includes aids to encourage patient medication compliance, [48] which can also be incorporated into these disease specialties [49]. Some patient apps currently on the market [50] include features that allow patients to scan barcodes and obtain more health-related information [51] to help highlight side-effects of medication and/ or contraindications [52].

Through some apps, patients who subscribe to a system can login health concerns about prescribed medication [47-52]. There are also web portals that can help highlight when a patient could consult a specialist [53].

\section{The Role of Imaging and Screening}

Several authors have emphasized the importance of early screening in patients with diabetes mellitus (T2DM) [15-18,54-58]. Patients with an eGFR below $60 \mathrm{~mL} / \mathrm{min} / 1.73$ $\mathrm{m}^{2}$ (stages 3-5 CKD) are at increased risk of CVD in comparison to the general population [11]. Between 20092010 , CKD accounted for $1.3 \%$ of the UK health care budget directly and indirectly up to $25 \%$ of the healthcare budget in the United States in patients aged over 65 [56].

Of note, the role of imaging in correlation with serum biomarkers of diabetes has been investigated in renal diabetic patients [56]. In a study which explored imaging, upon 42 diabetic patients ( 28 males, 14 females; mean age $=33$ years) and 17 age-and sex-matched volunteers. Diabetic patients were normalbuminuric $(n=27)$ and macroalbuminuric $(n=15)$. Patients and volunteers underwent diffusion tensor imaging of kidneys. There was a significant difference on imaging results of the renal cortex in diabetic patients with macroalbuminuric versus normalbuminuric. This may be attributed to renal tubular damage, and interstitial renal fibrosis in patients with macroalbuminuria. Differentiation of patients with macroalbuminuria versus normalbuminuric is important; initial intervention and novel drug therapy prescribed to patients with microalbuminuria could preclude CVD risk and overall reduced renal function [56].

Where identifying CVD risk is concerned, different reporting systems in medical imaging have introduced uniform documenting linked with information controlling patient care (Abdel et al. 2018). The Coronary Artery Disease Reporting and Data System (CAD-RADS TM) is a classification system suggested by the Society of Cardiovascular Computed Tomography, which has been used to establish coronary artery stenosis severity [57].
In one study coronary artery severity implementing CADRADS, was used to assess the degree of stenosis in patients with stable chest pain. 96 patients (82 male, 14 female, age range 45-74 years, mean age 63 years) who underwent coronary imaging. The authors inform providing a single, perpatient severity score for CVD suspect/high-risk patients would increase the clarity in reporting and recommendation for the management of coronary artery stenosis [57]. This would also be proactive in the care of renal patients, since imaging is needed to make critical health decisions to support diagnoses and treatment.

\section{Discussion}

There is a continuous need to distribute health advice to renal patients $[54,55]$. Physical activity and stress are entities that need further investigation to preclude cardiovascular complications in renal patients. It is also paramount that nephrologists and cardiologists advise patients to live active lifestyles [56-58].

Renal patients should be advised to reach good blood pressure and cholesterol levels; to abstain from smoking, to reduce excess body weight and participate in routine exercise [56-76]. A recent review explores physical activity and elucidation of its effects on biochemical and haematological parameters in haemodialysis patients [74]. Intervention research is needed to understand how such parameters are affected in patients post renal transplant.

Intervention research to explore whether technology and a social media-based (WeChat) programme can facilitate risk factor monitoring and self-management has been impressive [77] to improve outcomes in patients with CHD. A systematic review and meta-analyses assess the effectiveness of app-based interventions, to promote lifestyle changes in patients with Noncommunicable Diseases (NCDs) is also striking. Of the 1588 records examined, 7 studies included diabetes patients only, 1 study included heart patients only, and 1 study included both diabetes and heart patients. Authors inform research with long-term follow-up is needed to assess effect of technology for other NCDs long-term conditions [37].

CVD complications can be both acute and chronic. Health awareness and education is still a much-needed resource for the renal patient; more awareness and understanding of CVD complications need to be proactively implemented as part of a patient's care plan [58]. It is believed acute more collaboration between cardiology and nephrology specialties are required to combat CVD complications.

\section{Conclusion}

A complete list of CVD complications has not been formulated; indeed, they extend the scope of this review. Surgical interventions are also on the increase; surgery should be a last resort and not another 'usual' mode of prevention and intervention for CVD impediment. CVD risks increase owing to long-term complications in renal patients whether on a form of RRT or following renal transplant [58,78,79]. 
Whilst prescribing renal patients anti-hypertensives is advantageous, the side-effects add to CKD burden $[41,78,79]$. Long-term RRT protocols also contribute to health complications. There is a need for more robust therapeutic clinical trials and/or intervention research integrating knowledge base from social health sciences to preclude CVD complications in renal patients.

\section{Summary-What this study adds}

Measuring endothelial function, providing nutrition and physical activity advice to prevent CVD and MI risks are just a few traditional interventional measures [58,78,79]; innovative screening, imaging and technology can also assist patients and teams [48-53]. Compared to later reviews surrounding this subject, this paper highlights given that patients are being prompted more to self-care through technology, now more than ever there needs to be a closer link between randomized clinical trials between the cardio-nephrology specialties, and social sciences to inform how best to prevent patients with CKD developing CVD events.

\section{References}

1. Hancock WW, Lu B, Gao W, et al. Requirement of the chemokine receptor CXCR3 for acute allograft rejection. J Exp Med. 2000;192(10):1515-20.

2. Inston NG, Cockwell P. The Evolving Role of Chemokines and their Receptors in Acute Allograft Rejection. Nephrol Dial Transplant. 2002;17:1374-79.

3. Yellon DM, Alkhulaifi AM, Pugsley WB. Preconditioning the human myocardium. Lancet. 1993;342(8866):276-7V.

4. Bolte CS, Liao S, Gross GJ, et al. Remote preconditioning-endocrine factors in organ protection against ischemic injury. Endocr Metab Immune Disord Drug Targets. 2007;7(3):167-75.

5. Loukogeorgakis SP, Panagiotidou AT, Broadhead MW, et al. Remote ischemic preconditioning provides early and late protection against endothelial ischemia-reperfusion injury in humans: role of the autonomic nervous system. J Am Coll Cardiol. 2005;46(3):450-6.

6. Loukogeorgakis SP, Williams R, Panagiotidou AT, et al. Transient limb ischemia induces remote preconditioning and remote postconditioning in humans by a K(ATP)channel dependent mechanism. Circulation. 2007;116(12): 1386-95.

7. Jhee JH, Park J, Kim H, et al. The Optimal Blood Pressure Target in Different Dialysis Populations. Sci Rep. 2018;8(1):14123.

8. Kerr M, Bray B, Medcalf J, et al. Estimating the financial cost of chronic kidney disease to the NHS in England. Nephrol Dial Transplant. 2012;27 Suppl 3:iii73-80.

9. Stoumpos S, Jardine AG, Mark PB. Cardiovascular morbidity and mortality after kidney transplantation. Transpl Int. 2015;28(1):10-21.

10. Ventura HO, Mehra MR. Improvement of heart failure after renal transplantation: the complex maze of cardiorenal interaction. J Am Coll Cardiol. 2005;45(7):1061-3.
11. https://www.england.nhs.uk/improvement-hub/wpcontent/uploads/sites/44/2017/11/Chronic-KidneyDisease-in-England-The-Human-and-Financial-Cost.pdf

12. https://www.nice.org.uk/about/nice-communities/publicinvolvement/your-care

13. Anavekar NS, McMurray JJ, Velazquez EJ, et al. Relation between renal dysfunction and cardiovascular outcomes after myocardial infarction. N Engl J Med. 2004;351(13): 1285-95.

14. McIntyre NJ, Fluck R, McIntyre C, et al. Treatment needs and diagnosis awareness in primary care patients with chronic kidney disease. Br J Gen Pract. 2012;62(597):e227-32.

15. Tonelli M, Muntner P, Lloyd A, et al. Using proteinuria and estimated glomerular filtration rate to classify risk in patients with chronic kidney disease: a cohort study. Ann Intern Med. 2011;154(1):12-21.

16. Collins AJ, Chen SC, Gilbertson DT, et al. CKD surveillance using administrative data: impact on the health care system. Am J Kidney Dis. 2009;53(3 Suppl 3):S27-36.

17. Levey AS, Coresh J, Balk E, et al. National Kidney Foundation practice guidelines for chronic kidney disease: evaluation, classification, and stratification. Ann Intern Med. 2003;139(2):137-47.

18. Mickan S, Tilson JK, Atherton $\mathrm{H}$, et al. Evidence of effectiveness of health care professionals using handheld computers: a scoping review of systematic reviews. J Med Internet Res. 2013;15(10):e212.

19. https://www.renalreg.org/reports/2015-eighteenth-annualreport/

20. Dolgos S, Hartmann A, Jenssen T, et al. Determinants of short-term changes in body composition following renal transplantation. Scand J Urol Nephrol. 2009;43(1):76-83.

21. Dolgos S, Hartmann A, Bollerslev J, et al. The importance of body composition and dry weight assessments in patients with chronic kidney disease. Acta Physiol Hung. 2011;98(2):105-16.

22. Armstrong KA, Campbell SB, Hawley CM, et al. Impact of obesity on renal transplant outcomes. Nephrology. 2005;10(4):405-13.

23. Ojo OA. Cardiovascular complications after renal transplantation and their prevention. Transplantation. 2006;82(5):603-11.

24. Silkensen JR. Long-Term Complications in Renal Transplantation. J Am Soc Nephrol. 2000;11(3):582-8.

25. Hancock WW, Gao W, Csizmadia V, et al. Donor-derived IP-10 initiates development of acute allograft rejection. J Exp Med. 2001;193(8):975-80.

26. Sells RA. Cardiovascular complications following renal transplantation. Transplantation Reviews. 1997;11(3): 111-26.

27. Mojto V, Gvozdjakova A, Kucharska J, et al. Effects of complete water fasting and regeneration diet on kidney function, oxidative stress and antioxidants. Bratisl Lek Listy. 2018;119(2):107-11. 
Citation: Muhammad SN. Cardio-Nephrology: The need for more collaborative efforts between therapeutic clinical trials and social health sciences to preclude cardiovascular disease complications in renal patients. J Clin Nephrol Ther 2019;3(1):3-9.

28. Gutierrez-Mariscal FM, Yubero-Serrano EM, Villalba JM, et al. Coenzyme $\mathrm{Q}(10)$ : From bench to clinic in aging diseases, a translational review. Crit Rev Food Sci Nutr. 2018;16:1-18.

29. Mortensen SA, Rosenfeldt F, Kumar A, et al. The effect of CoQ10 on morbidity and mortality in chronic heart failure: the Q-SYMBIO randomized double blind trial. JACC Heart Failure. 2014;2(6): 641-9.

30. Montresor A, Toffali L, Constantin G, et al. Chemokines and the signalling modules regulating integrin affinity. Front Immunol. 2012;3:127.

31. Sternberg EM. Neuroendocrine Regulation of Autoimmune/ Inflammatory Disease. J Endocrinol. 2001;169(3):429-35.

32. Segerer S, Nelson PJ, Schlöndorff D. Chemokines, chemokine receptors, and renal disease: from basic science to pathophysiologic and therapeutic studies. J Am Soc Nephrol. 2000;11(1):152-76.

33. Muhammad S. The Immune System, Transplant Rejection and Psychological Stress. United Kingdom:LAP Publishing; 2014.

34. Smart N, Steele M. Exercise training in haemodialysis patients: A systematic review and meta-analysis. Nephrology (Carlton). 2011;16(7):626-32.

35. Ma KW, Greene EL, Raij L. Cardiovascular Risk Factors in Chronic Renal Failure and Haemodialysis Populations. Am J Kidney Dis. 1992;19(6):505-13.

36. Smith MC, Dunn MJ. Hypertension in Renal Parenchymal Disease, in Laragh JH, Brenner BM (Eds): Hypotension, Pathophysiology, Diagnosis and Management. New York: Raven Press Limited;1990.

37. Lunde P, Nilsson BB, Bergland A, et al. The Effectiveness of Smartphone Apps for Lifestyle Improvement in Noncommunicable Diseases: Systematic Review and Meta-Analyses. J Med Internet Res. 2018;20(5):e162.

38. Lubas A, Kade G, Ryczek R, et al. Ultrasonic evaluation of renal cortex arterial area enables differentiation between hypertensive and glomerulonephritis-related chronic kidney disease. Int Urol Nephrol. 2017;49(9):1627-35.

39. Luo M, Guan X, Luczak ED, et al. Diabetes increases mortality after myocardial infarction by oxidizing CaMKII. J Clin Invest. 2013;123(3):1262-74.

40. Junttila MJ, Kiviniemi AM, Lepojärvi ES, et al. Type 2 diabetes and coronary artery disease:Preserved ejection fraction and sudden cardiac death. Heart Rhythm. 2018;15(10):1450-6.

41. Wan RK, Mark PB, Jardine AG. Cardiovascular disease management in renal transplant recipients: more or less treatment? Transplantation. 2006;82(6):737-8.

42. Wan RK, Mark PB, Jardine AG. The cholesterol paradox is flawed; cholesterol must be lowered in dialysis patients. Semin Dial. 2007;20(6):504-9.

43. Joss N, Staatz CE, Thomson AH, et al. Predictors of new onset diabetes after renal transplantation. Clin Transplant. 2007;21(1):136-43.

44. Akman B, Uyar M, Afsar B, et al. Lipid Profile During Azathioprine or Mycophenolate Mofetil Combinations with Cyclosporine and Steroids. Transplant Proc. 2007;39(1):135-7.

45. Geddes CC, Jardine AG, Kingsmore D, et al. Five-year outcomes after a change from a cyclosporin-based to a 'low-dose' tacrolimus-based primary immunosuppression regimen for incident kidney transplants--the Glasgow experience. Clin Transpl. 2012:95-102.

46. http://www.wales.nhs.uk/sites3/documents/434/ Workforce.pdf

47. Muhammad S, Allan M, Ali F, et al. The renal patient support group: supporting patients with chronic kidney disease through social media. J Ren Care. 2014;40(3): 216-8.

48. Albano L, Casez JP, Bekri S, et al. Effects of tacrolimus vs cyclosporin-A on bone metabolism after kidney transplantation: a cross-sectional study in 28 patients. Nephrol Ther. 2005;1(2):115-20.

49. Adams DH, Sanchez-Fueyo A, Samuel D. From immunosuppression to tolerance. J Hepatol. 2015;62(1 Suppl):S170-85.

50. Jardine AG. Assessing the Relative Risk of Cardiovascular Disease among Renal Transplant Patients receiving Tacrolimus or Cyclosporine. Transpl Int. 2005;18(4): 379-84.

51. Gameiro J, Gonçalves M, Pereira M, et al. Obesity, acute kidney injury and mortality in patients with sepsis: a cohort analysis. Ren Fail. 2018;40(1):120-6.

52. Bottomley MJ, Harden PN. Update on the long-term complications of renal transplantation. $\mathrm{Br}$ Med Bull. 2013;106:117-34.

53. O'Donoghue KJM, Reed RD, Knight SR, et al. Systematic review of clinical practice guidelines in kidney transplantation. BJS Open. 2017;1(4):97-105.

54. Marks A, Macleod C, McAteer A, et al. Chronic kidney disease, a useful trigger for proactive primary care? Mortality results from a large U.K. cohort. Fam Pract. 2013;30(3):282-9.

55. Mason P. Basic Concepts of Clinical Testing. Pharmaceutical Journal. 2004;272: 384-6.

56. Abdel Razek A, Al-Adlany M, Alhadidy A, et al. Diffusion tensor imaging of the renal cortex in diabetic patients: Correlation with urinary and serum biomarkers. Abdom Radiol (NY). 2017;42(5):1493-1500.

57. Abdel Razek AAK, Elrakhawy MM, Yossof MM, et al. Inter-observer agreement of the Coronary Artery Disease Reporting and Data System (CAD-RADS (TM)) in patients with stable chest pain. Pol J Radiol. 2018;83: e151-9.

58. Nelson EC, Eftimovska E, Lind C, et al. Patient reported outcome measures in practice. BMJ. 2015;350:g7818.

59. Musso G, Cassader M, Cohney S, et al. Fatty Liver and Chronic Kidney Disease: Novel Mechanistic Insights and Therapeutic Opportunities. Diabetes Care. 2016;39(10): 1830-45.

60. van Gemst JJ, Kouwenberg M, Rops ALWMM, et al. Differential binding of chemokines CXCL1, CXCL2 and CCL2 to mouse glomerular endothelial cells reveals 
specificity for distinct heparan sulfate domains. PLoS One. 2018;13(9):e0201560.

61. Dayer L, Heldenbrand S, Anderson P, et al. Smartphone medication adherence apps: potential benefits to patients and providers: response to Aungst. J Am Pharm Asso. 2013;53(4):345.

62. Dayer L, Heldenbrand S, Anderson P, et al. Smartphone medication adherence apps: potential benefits to patients and providers. J Am Pharm Assoc (2003). 2013;53(2): $172-81$.

63. Santo K, Chow CK, Thiagalingam A, et al. MEDication reminder APPs to improve medication adherence in Coronary Heart Disease (MedApp-CHD) Study: a randomised controlled trial protocol. BMJ Open. 2017;7:e017540.

64. Reddy R, Majmudar M, Dhopeshwarkar N, et al. Mobile health apps preferences and practice among ambulatory cardiovascular patients. Future Cardiol. 2018;14(5):381-8.

65. Elavsky S, Smahel D, Machackova H. Who are mobile app users from healthy lifestyle websites? Analysis of patterns of app use and user characteristics. Transl Behav Med. 2017;7(4):891-901.

66. DiFilippo KN, Huang WH, Andrade JE, et al. The use of mobile apps to improve nutrition outcomes: A systematic literature review. J Telemed Telecare. 2015;21(5):243-53.

67. de Jongh T, Gurol-Urganci I, Vodopivec-Jamsek V, et al. Mobile phone messaging for facilitating self-management of long-term illnesses. Cochrane Database Syst Rev. 2012;12:CD007459.

68. Vodopivec-Jamsek V, de Jongh T, Gurol-Urganci I, et al. Mobile phone messaging for preventive health care. Cochrane Database Syst Rev. 2012;12:CD007457.

69. Menke J, Zeller GC, Kikawada E, et al. CXCL9, but not CXCL10, promotes CXCR3-dependent immune-mediated kidney disease. J Am Soc Nephrol. 2008;19(6):1177-89.

70. Fischereder M, Kretzler M. New immunosuppressive strategies in renal transplant recipients. J Nephrol. 2004;17(1):9-18.

71. Shephard RJ, Rhind S, Shek PN. Exercise and training: influences on cytotoxicity, interleukin-1, interleukin-2 and receptor structures. Int J Sports Med. 1994;15 Suppl 3:S154-66.
72. Koufaki P, Greenwood S, Painter P, et al. The BASES expert statement on exercise therapy for people with chronic kidney disease. J Sports Sci. 2015;33(18):1902-7.

73. Koufaki P, Kouidi E. Current best evidence recommendations on measurement and interpretation of physical function in patients with chronic kidney disease. Sports Med. 2010;40(12):1055-74.

74. Muhammad S. End-Stage Renal Disease (ESRD): Physical Activity and Elucidation of its Effects on Biochemical \& Haematological Parameters in Haemodialysis Patients. Ann Clin Lab Res. 2016;4:3.

75. Culleton BF, Hemmelgarn BR. The clinical epidemiology of cardiovascular diseases in chronic kidney disease: Is Chronic Kidney Disease a Cardiovascular Disease Risk Factor? Seminars in dialysis. 2003;16(2)95.

76. Johansen KL, Sakkas GK, Doyle J, et al. Exercise counseling practices among nephrologists caring for patients on dialysis. Am J Kidney Dis. 2003;41(1):171-8.

77. Dorje T, Zhao G, Scheer A, et al. SMARTphone and social media-based Cardiac Rehabilitation and Secondary Prevention (SMART-CR/SP) for patients with coronary heart disease in China: a randomised controlled trial protocol. BMJ Open. 2018;8(6): e021908.

78. Heiwe S, Jacobson SH. Exercise training for adults with chronic kidney disease. Cochrane Database Syst Rev. 2011;(10):CD003236.

79. Painter P, Roshanravan B. The association of physical activity and physical function with clinical outcomes in adults with chronic kidney disease. Curr Opin Nephrol Hypertens. 2013;22(6):615-23.

\section{*Correspondence to}

Mr. Shahid Nazir Muhammad,

Co-founder and Head of Research,

Department of Research and Development,

The Renal Patient Support Group (RPSG), England UK,

E-mail: rpsg1@outlook.com 